OPEN ACCESS

Edited by:

David Fuller,

University of Florida, USA

Reviewed by:

Silvia Pagliardini,

University of Alberta, Canada

Mai EIMallah,

University of Massachusetts Medical

School, USA

*Correspondence:

E. Fiona Bailey

ebailey@email.arizona.edu

Specialty section:

This article was submitted to

Respiratory Physiology,

a section of the journal

Frontiers in Physiology

Received: 02 September 2016 Accepted: 11 January 2017 Published: 25 January 2017

Citation:

LaCross A, Watson PJ and Bailey EF (2017) Association between Laryngeal Airway Aperture and the Discharge Rates of Genioglossus Motor Units.

Front. Physiol. 8:27.

doi: 10.3389/fphys.2017.00027

\section{Association between Laryngeal Airway Aperture and the Discharge Rates of Genioglossus Motor Units}

\author{
Amy LaCross ${ }^{1}$, Peter J. Watson ${ }^{2}$ and E. Fiona Bailey ${ }^{1 *}$ \\ ${ }^{1}$ Department of Physiology, College of Medicine, University of Arizona, Tucson, AZ, USA, ${ }^{2}$ Department of Speech, \\ Language, and Hearing Science, College of Liberal Arts, University of Minnesota, Minneapolis, MN, USA
}

We know very little about how muscles and motor units in one region of the upper airway are impacted by adjustments in an adjacent airway region. In this case, the focus is on regulation of the expiratory airstream by the larynx and how changes in laryngeal aperture impact muscle motor unit activities downstream in the pharynx. We selected sound production as a framework for study as it requires (i) sustained expiratory airflow, (ii) laryngeal airway regulation for production of whisper and voice, and (iii) pharyngeal airway regulation for production of different vowel sounds. We used these features as the means of manipulating expiratory airflow, pharyngeal, and laryngeal airway opening to compare the effect of each on the activation of genioglossus (GG) muscle motor units in the pharynx. We show that some GG muscle motor units (a) discharge stably on expiration associated with production of vowel sounds, (b) are exquisitely sensitive to subtle alterations in laryngeal airflow, and (c) discharge at higher firing rates in high flow vs. low flow conditions even when producing the same vowel sound. Our results reveal subtle changes in GG motor unit discharge rates that correlate with changes imposed at the larynx, and which may contribute to the regulation of the expiratory airstream.

Keywords: genioglossus, motor unit, phonation

\section{INTRODUCTION}

Human tongue muscles participate in respiration-related and voluntary movements. In regard to respiration, it is evident that the extrinsic tongue protrudor muscle genioglossus (GG) defends the airway against inspiratory narrowing at rest (Cheng et al., 2011), in exercise (Walls et al., 2013), and during sleep (Chuang et al., 2009). Much less is known of the GG's role in regulating airway lumen in activities that depend on control of expiratory airflow including coughing, speaking, wind instrument playing, and singing.

Sustained expirations that are the hallmark of conversational speech result from passive and active forces that operate on the chest wall (ribcage + abdomen; Grimby et al., 1968; Bunn and Mead, 1971; Agostoni et al., 1979) and active regulation of downstream resistances (Remmers and Bartlett, 1977; England et al., 1982; Giering and Daubenspeck, 1990). Indeed, for sound generating behaviors, structures such as the larynx (Finnegan et al., 2000; Gillespie et al., 2015), nose (Peters and Boves, 1988; Sapienza et al., 1997), and velopharynx (Warren, 1986; Warren et al., 1989) fulfill dual functions serving as variable resistors that regulate air pressure and airflow and as sites where the vowels and consonants of language are formed. 
The process by which airway resistors are controlled and coordinated is of fundamental interest and importance both to speech and to respiratory motor control. Whereas, most previous research has examined the GG's role defending the airway on inspiration (Remmers et al., 1978; Mezzanotte et al., 1992, 1996; Fogel et al., 2001; Remmers, 2001), in this case we look for evidence of its expiration-related activity. Recently we documented expiration-related GG activity during moderate and heavy exercise that suggested a role for the muscle in the regulation of the expiratory airstream (Walls et al., 2013). In that circumstance, we hypothesized expiration-related GG activity is modulated in parallel with laryngeal airway aperture (England and Bartlett, 1982) to dilate the airway to reduce expiratory time. Here we explore the possibility further asking how changes imposed at the larynx in the process of speech communication, affect the downstream activation of genioglossus muscle motor units. Accordingly, we exclude from our analysis inspiratory motor unit activity (Figure 1A) and sustained or tonic motor unit activity (Figure 1B), electing to focus in this case on GG motor unit activity associated exclusively with the production of sound on expiration (Figure 1C).

\section{METHODS}

\section{Participants}

Thirty-one adults (21 females and 10 males; mean age \pm SD, 20-24 years) participated in the study. Only healthy participants $\left(\mathrm{BMI} \pm \mathrm{SD}, 22.69 \pm 3.17 \mathrm{~kg} \cdot \mathrm{m}^{2}\right)$ were recruited to the study. Adults who reported a history of respiratory disease or impairment, major surgery, or injury involving the upper airway or respiratory or sound production systems were excluded. All were native speakers of American English. The Human Subjects Committee at the University of Arizona approved all experimental procedures and subjects provided written, informed consent prior to participation.

\section{Experimental Conditions Laryngeal Airway Manipulations}

In this study, subjects were required to produce two forms of sound energy (a) voice (phonation) and (b) whisper. In a third condition, subjects used an artificial sound source or electrolarynx. An explanation of each of these conditions and the rationale for their inclusion is provided below. The reader is directed to the experimental schematic provided in Figure 3 and the accompanying legend.

1. Phonation. Voice is produced when expiratory airflow from the lungs sets the vocal folds into vibration converting aerodynamic power into sound energy (e.g., acoustic power; Simonyan and Horwitz, 2011). Subjects were instructed to speak/produce sound at their normal conversational loudness (Seashore, 1938). In this condition, vocal folds approximate and create a resistance to the expiratory airstream that gives rise to a complex periodic sound energy.

2. Whisper. Subjects were instructed to imagine whispering in someone's ear. In this condition, vocal folds approximate the midline forming a glottis that is V or Y shaped (Solomon et al.,
1989; Sundberg et al., 2010) that creates turbulent airflow and complex aperiodic sound energy (Monoson and Zemlin, 1984; Solomon et al., 1989; Matsuda and Kasuya, 1999). Expiratory airflow in this condition is higher and more turbulent than for phonation (Schwartz, 1968; Weismer and Longstreth, 1980; Stathopoulos et al., 1991).

3. Electro-larynx condition. This condition served as a control condition. The electro-larynx is a battery-powered device coupled to a small tube which, when placed in the corner of the mouth, introduces mechanically generated complex and periodic sound energy into the vocal tract. Because the EL is the sound source there is no requirement for vocal cord adduction or vibration as is the case for whisper and voice. Rather, subjects continue to breathe normally without interrupting sound production. Expiratory airflow is at its nadir in this condition.

\section{Pharyngeal Airway Manipulations}

The sound energy produced at the larynx is filtered and amplified as it passes through the upper airway. The sound produced by the larynx resonates in the chambers formed by the pharynx, nasal and oral cavities creating the sounds we recognize as vowels. Importantly, the dimensions of the pharyngeal airway are determined in large part by the tongue and changes in tongue placement are required to achieve each different vowel and to shift from vowel production to resting breathing (see Figures $2 \mathrm{Ai}$-ii). Thus, each vowel is the result of a distinct pharyngeal airway geometry. We asked participants to say the vowels /i/, /æ/, /u/, /a/ as pronounced in the words; heat, hat, hoot and hot (Figure 2Aii). Subjects sustained each vowel for $1-$ $2 \mathrm{~s}$ and completed 6-8 repetitions per trial. There were no time constraints for initiation or completion of the task.

Audio signals were recorded with the highest digital sampling rate available when multiplexed with the digitized physiologic (motor unit) data. The audio signal was recorded $(16.667 \mathrm{kHz}$ sampling frequency; 16 bit depth) via head-mounted microphone (Opus 55.18 MK II, BeyerDynamic, Long Branch, NJ) positioned $2 \mathrm{~mm}$ from the left corner of the mouth. To eliminate aliasing effects, the speech signal was filtered using a 10th-order variable low-pass filter unit (32 kHz, 4301, CED, Cambridge, UK) before transmission to a data acquisition interface (Power1401, CED, Cambridge, UK). F1 and F2 formant frequencies were identified for each utterance by visual examination of the spectrographic display in Praat (Version 5.3.14) and determined to be comparable to previously reported values in men and women (Peterson and Barney, 1952; Hillenbrand et al., 1995).

Chest wall motions were monitored via strain-gauge transducers (Pneumotrace, UFI, Morro Bay, CA) positioned around the thorax at the mid-sternal level and around the abdomen at the level of the umbilicus. Output from these sensors reflected changes in circumference of the rib cage and abdomen and were used to distinguish inspiration and expiration.

\section{Magnetic Resonance Imaging (MRI)}

In this case, we obtained images of the upper airway in four subjects (two male and two female) Representative images obtained from one subject are presented in Figure 2. We include 


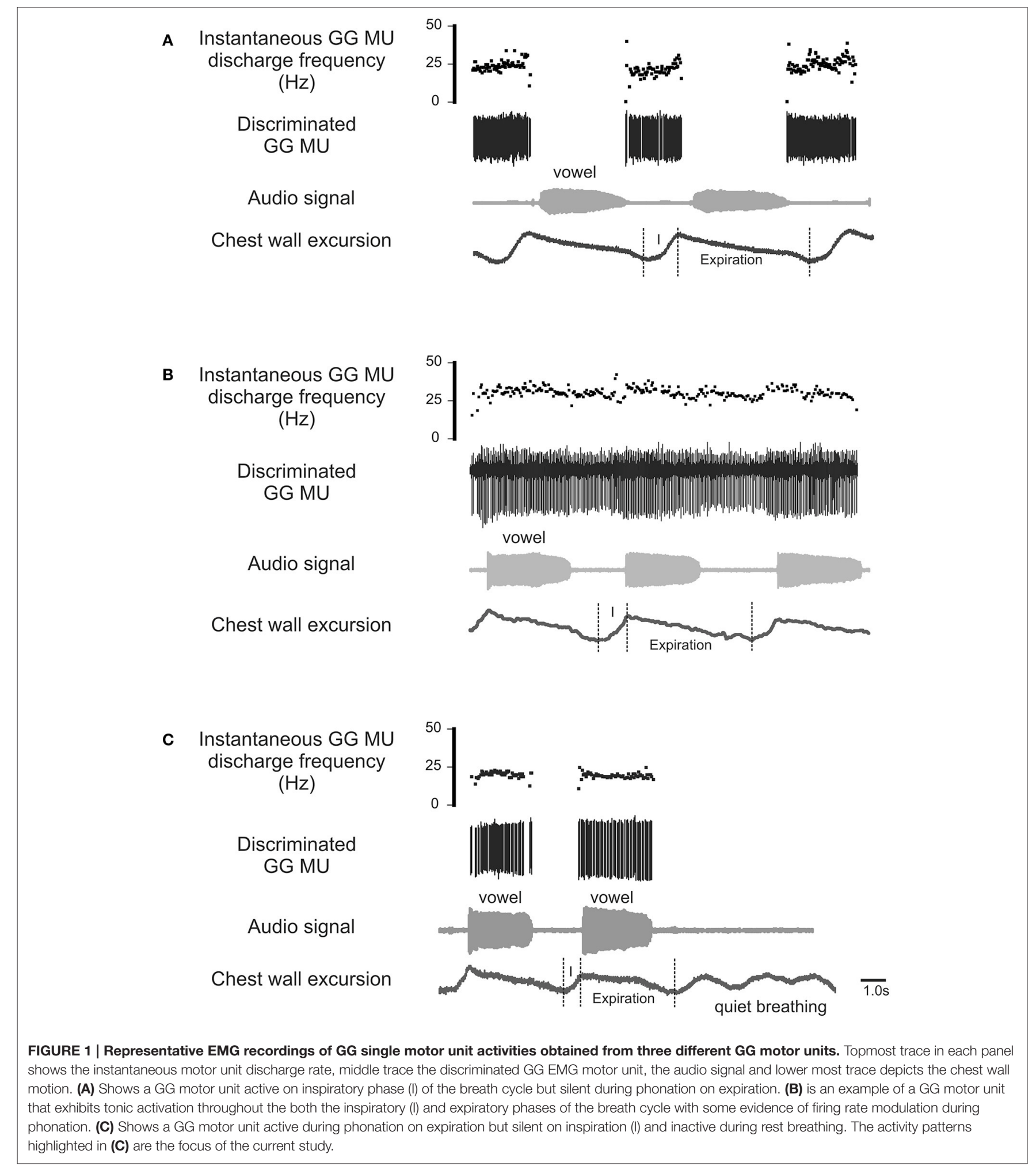

these images to make clear the configuration of the pharyngeal airway in the production of of $/ \mathrm{i} /, / \Re /, / \mathrm{u} /, / \mathrm{a} /$. Images were obtained using a Siemens Magnetom Skyra 3 Tesla MRI Scanner (University of Arizona, MRI Facility) and subjects lay supine in the scanner and received instructions from the experimenter via headphones prior to each production. Note that audio and motor unit recordings were not attempted during imaging sequences. 
Ai

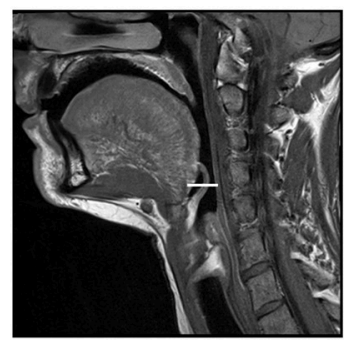

$\mathrm{Bi}$

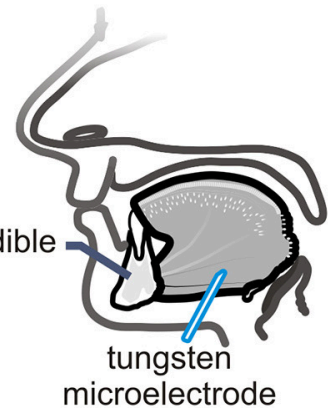

Aii
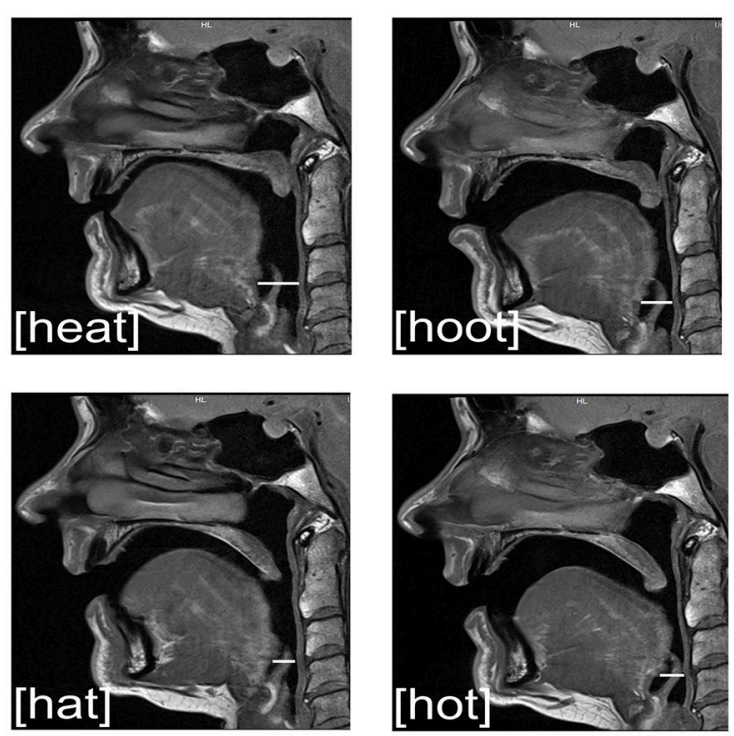

Bii
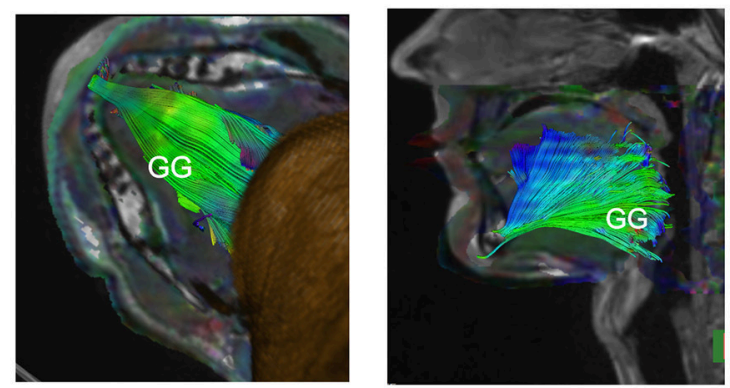

FIGURE 2 | (A) Sagittal MRls obtained from one subject during rest breathing and production of vowel sounds. (Ai) At left, the position of the tongue and the pharyngeal and oral airway lumens in quiet breathing. (Aii) Shows pharyngeal and oral airway lumens in production each of the vowels /i/, /æ/, /u/, /a/ as heard in the words /heat/, /hat/, /hoot/, and /hot/. Note that the dimensions of the pharyngeal airway lumen (horizontal white line in each panel) are determined by the position of the tongue which differs somewhat for each vowel. The pharyngeal airway lumen is greater for the vowels in /heat/ and /hoot/ relative to /hat/ and /hot/ and relative to quiet breathing. (Bi) Schematic of the experimental set up showing approximate location of the recording electrode within the horizontal compartment of the GG. (Bii) A representative DT image from a subject showing muscle fiber tracts superimposed on MR images (low $B$-value magnitude images). This inferior view of the floor of mouth reveals the horizontal course of GG muscle fibers. EMG recordings were made within the GG muscle $\sim 1.0-3.0 \mathrm{~cm}$ posterior to the mandible in muscle fibers that extend in a horizontal plane from the mental symphysis of the mandible anteriorly to the tongue base, in the region of the epiglottis, posteriorly. Activation of this compartment of the GG results in forward motion of the tongue and increased pharyngeal airway lumen.

Sagittal, axial and coronal planes were imaged (on all planes: TE: $10 \mathrm{~ms}$, TR: $1500 \mathrm{~ms}$, ETL: $5 \mathrm{~ms}$, FOV: $150 \mathrm{~cm}$, Flip angle: $160^{\circ}$ ). For all scan types, an anterior neck coil (four element Flex coil, München, Germany) was used so that the desired portion of the head and neck were brought into the scanning field of view. To image GG muscle fibers for each vowel, a series of fifteen 3-mm thick contiguous, parallel, sagittal sections were gathered in an interleaved acquisition. The image set extended from just above the sinus cavities to the inferior border of the mandible.

\section{Probabilistic Diffusion Tractography}

We performed this additional analysis of imaged tissues to identify muscle fiber course. Tractography detects the direction of water diffusion and generates tracts across voxels yielding information about muscle fiber orientation (Gilbert et al., 2006).
Tracts were extended and connected across voxels only when they met an angular threshold criterion $<30^{\circ}$ (Siemens Neuro 3D software, University of Arizona). In this case we used tractography solely to highlight the orientation and trajectory (Gilbert et al., 2006) of those GG muscle fibers in the posterior tongue that contribute to airway dilation (Miyawaki et al., 1975; Buchaillard et al., 2009; Cheng et al., 2011). Example images obtained using diffusion tractography in the same subject are presented in Figure 2Bii.

\section{Electromyographic (EMG) Activity}

Motor unit recordings were obtained from the horizontal compartment of the GG using single tungsten microelectrodes (Frederick Haer, Bowdoinham, ME, 1-5 $\mu \mathrm{m}$ tip diameter, $10 \mathrm{M} \Omega$ at $1 \mathrm{KHz}$ ). Electrodes were inserted through the skin under the jaw into the mid-region of the GG muscle with entry points 


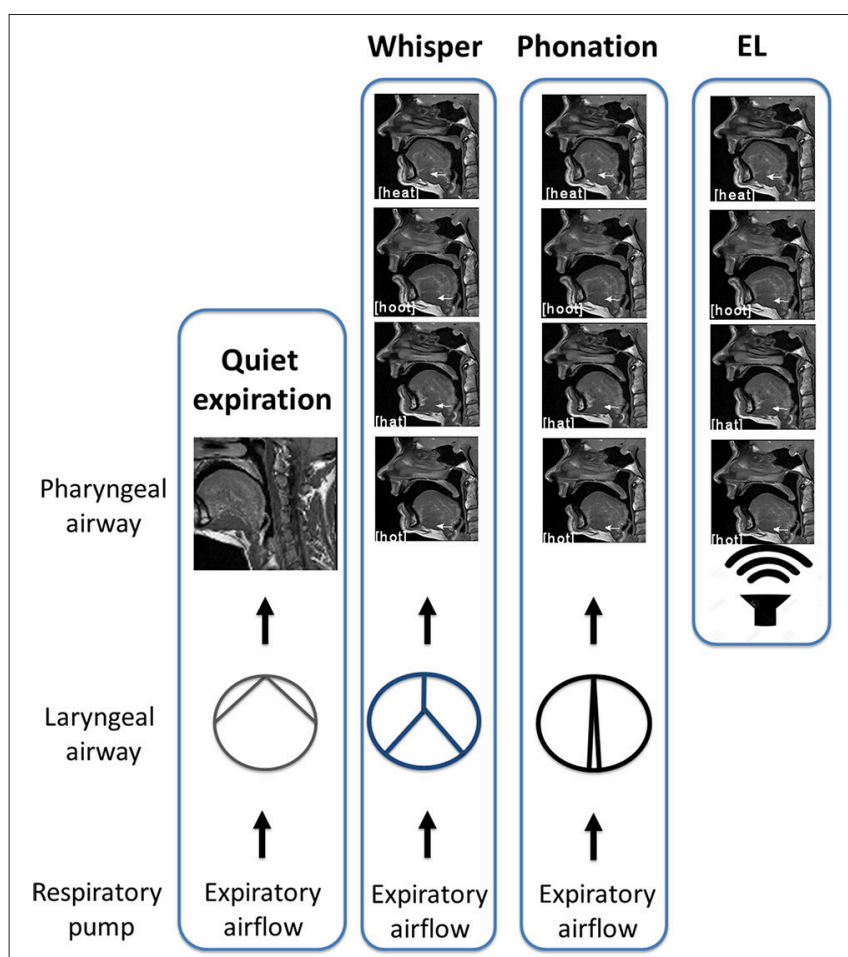

FIGURE 3 | Schematic of experimental conditions. In quiet breathing, air exits the lungs via the laryngeal airway where vocal cord adduction/abduction controls airway lumen and therefore, airflow. The laryngeal airway is widest for quiet expiration. In whisper, the vocal folds approximate the midline creating air turbulence. Turbulent airflow and vocal cord vibration result in complex aperiodic sound energy that is characteristic of whisper. When the vocal folds are more closely approximated, resistance to the expiratory airstream increases and results in a complex periodic sound energy that is characteristic of phonation. Shown at right is the "electro-larynx" condition. As the name suggests, the electro-larynx provides a battery-powered sound source can be introduced into the airway via a small tube placed in the corner of the mouth. In this condition, the electro-larynx not the larynx energizes the upper airway creating complex periodic sound energy for speech. As depicted, whereas whispered (top most panel), phonated (second panel) and electrolarynx productions of a vowel share the same tongue shape and position within the oral cavity the laryngeal configuration is distinct to that condition.

$\sim 1.5 \mathrm{~cm}$ from the midline and $\sim 2 \mathrm{~cm}$ posterior to the mandible (refer to Figure 2Bi). Tungsten electrodes cause little discomfort to the participant and do not require anesthetic of any kind. Depth of insertion was determined in advance via ultrasound (Pro Sound 3500, Aloka Co., Ltd., Tokyo, Japan). The depth from the skin surface to the GG varies with subject size and the depth to the inferior border of the muscle ranged from 1.5 to $2.5 \mathrm{~cm}$ from the skin surface (subjects $\mathrm{BMI} \pm \mathrm{SD}, 22.69 \pm$ $\left.3.17 \mathrm{~kg} \cdot \mathrm{m}^{2}\right)$. A surface electrode $(4.0 \mathrm{~mm}$ diameter $\mathrm{Ag}-\mathrm{AgCl})$ attached to the skin overlying the mastoid process served as the indifferent electrode and subjects were grounded via a $3 \mathrm{M}$ Red Dot electrode (Ag-AgCl) affixed to the skin overlying the clavicle. Motor unit action potentials were amplified (x1000), band-pass filtered (0.3-3 KHz; Grass Instruments, West Warwick, RI), and displayed on a storage oscilloscope to monitor size and shape of the impulses during data acquisition. Single motor unit activities were recorded using Spike2 data acquisition software (CED, Cambridge, UK).

\section{Data Analysis}

Analysis of motor unit action potentials was performed offline in Spike2 (CED, Cambridge, UK). Action potentials were discriminated using a template-matching algorithm based on waveform shape and amplitude and subsequently checked by visual inspection against the template unit waveform as discussed previously (Bailey et al., 2007). The mean instantaneous discharge rate was determined in the interval between utterance onset and offset, defined by the first and final zero-crossings of the audio waveform (refer Figure 1C). In determining average discharge rate and variability, only motor units for which activities could be followed throughout a series of vowel productions were included. For each recorded motor unit, average discharge rates were calculated from the average of three trials (each trial comprising 10-12 repetitions) of each vowel.

Statistical analyses were performed using SAS software (version 9.3). We used a linear-mixed ANOVA model to assess the effects of sex, laryngeal airway condition (phonation vs. whisper) and pharyngeal airway condition (each of four vowels) on motor unit discharge rates. Laryngeal and pharyngeal conditions were coded as fixed effects, subject and motor-unit (within subject) were coded as random effects. Note that of the total number of motor units recorded in whisper and phonation ( $n=116$ ), only $12 / 116$ were also recorded in the EL condition. Accordingly, the data from the EL condition were not included in the statistical analysis.

In the event of a significant $F$-value, differences were tested using post hoc comparisons with significance levels adjusted according to the Bonferroni procedure $(p=0.004)$. To analyze equality of variance of mean motor unit firing rates between vowel sounds, Levene's test of homogeneity was used to identify the variance of an individual motor unit's firing rate from the average firing rate for each vowel. Average firing rate was the dependent variable and vowel was the independent variable. Corrections for multiple comparisons were made using the Bonferroni procedure $(p=0.02)$.

\section{RESULTS}

We recorded the activities of 116 motor units in the region of the GG muscle that regulates the pharyngeal airway immediately downstream to the larynx in phonated and whispered productions of four vowels as follows; [i], [u], [æ], and $[a]$. The average number of motor units obtained from female $(4.1 \pm 4.2)$ and male participants $(3.1 \pm 2.09)$ was not different (Mann-Whitney $U=109.0, p=0.984$ ). The challenges of obtaining single motor unit recordings during speech sound production precluded us from obtaining equal numbers of recordings from all subjects however, the total number of motor units recorded for each vowel in whisper and phonation was comparable: [i]: $n=26,[\mathrm{u}]: n=34$, [æ]: $n=29$, and [a]: $n=27$.

Figure 4 shows representative recordings of the activities of a motor unit during production of the same vowel in each of the three conditions: (A) whisper, (B) phonation, and (C) electrolarynx. At right, time expanded views highlight subtle differences in motor unit activity between whispered, phonated, and electro-larynx productions of the vowel. Whereas, whispered 


\section{A Whisper}

\section{B Phonation}

\section{c Electrolarynx}

Audio Signal
GG
instantaneous
firing rate $(\mathrm{Hz})$
Discriminated
GG motor unit
GG
motor unit
Chest wall
signal

$\left.{ }_{0}^{50}\right]$

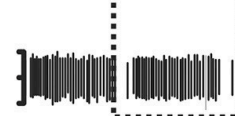

||||||||||||||||||||

\section{$\frac{\sqrt{ }}{5.0 \mathrm{~ms}}$}

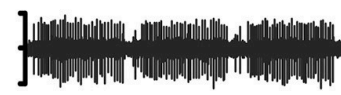

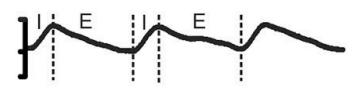

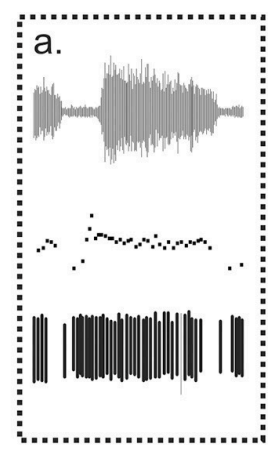

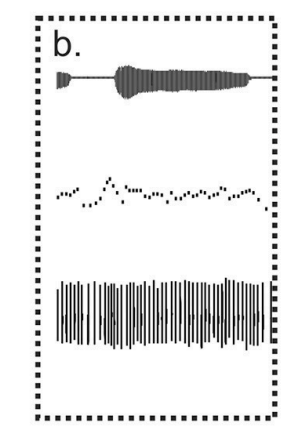

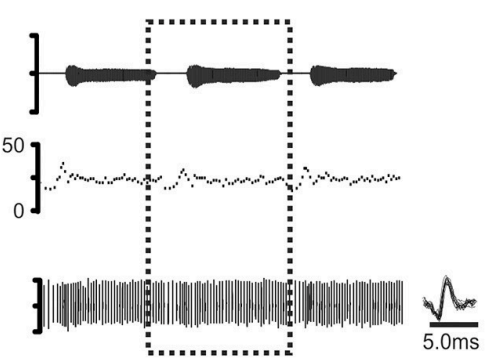

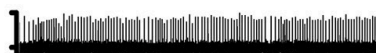

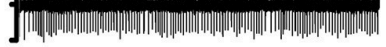

Chest wall signal

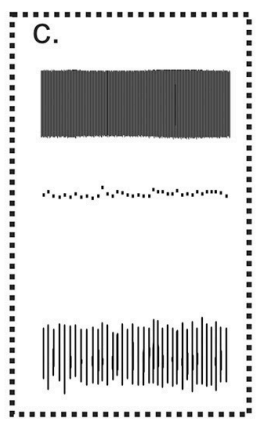

GG
motor unit
$\begin{gathered}\text { Chest wall } \\ \text { signal }\end{gathered}$
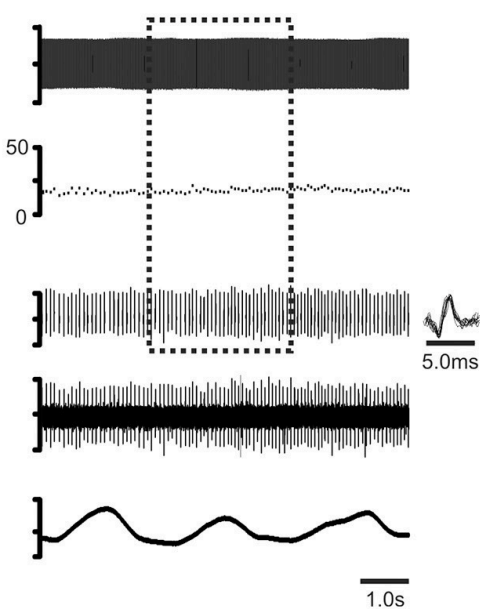

FIGURE 4 | Representative recordings of GG motor unit activity obtained from one subject in whisper (A), phonation (B) and electro-larynx (C) productions of the vowel /i/ as in /heat/. Topmost trace audio signal. Note that the greater amplitude audio signal in whisper (A) is the result of doubling the amplification in this condition relative to amplification settings for phonation and electro-larynx required to detect the speech signal in this condition. The middle three traces in each panel in show the untreated GG EMG signal, the discriminated GG single motor unit and the instantaneous motor unit discharge rate in each condition. Note that motor unit discharge averages in each task were determined in the expiratory (E) phase of each breath cycle in the interval between utterance onset and offset, defined by the first and final zero-crossings of the audio waveform. Note also, subtle differences in GG activity evident across the three conditions. Because tongue shape and position are presumed to remain stable to attain the target vowel, any between task differences in motor unit activity patterns are attributed to differences in valving of the expiratory airstream by the larynx that are characteristic of whisper, phonation and EL conditions (see Figure 3). 


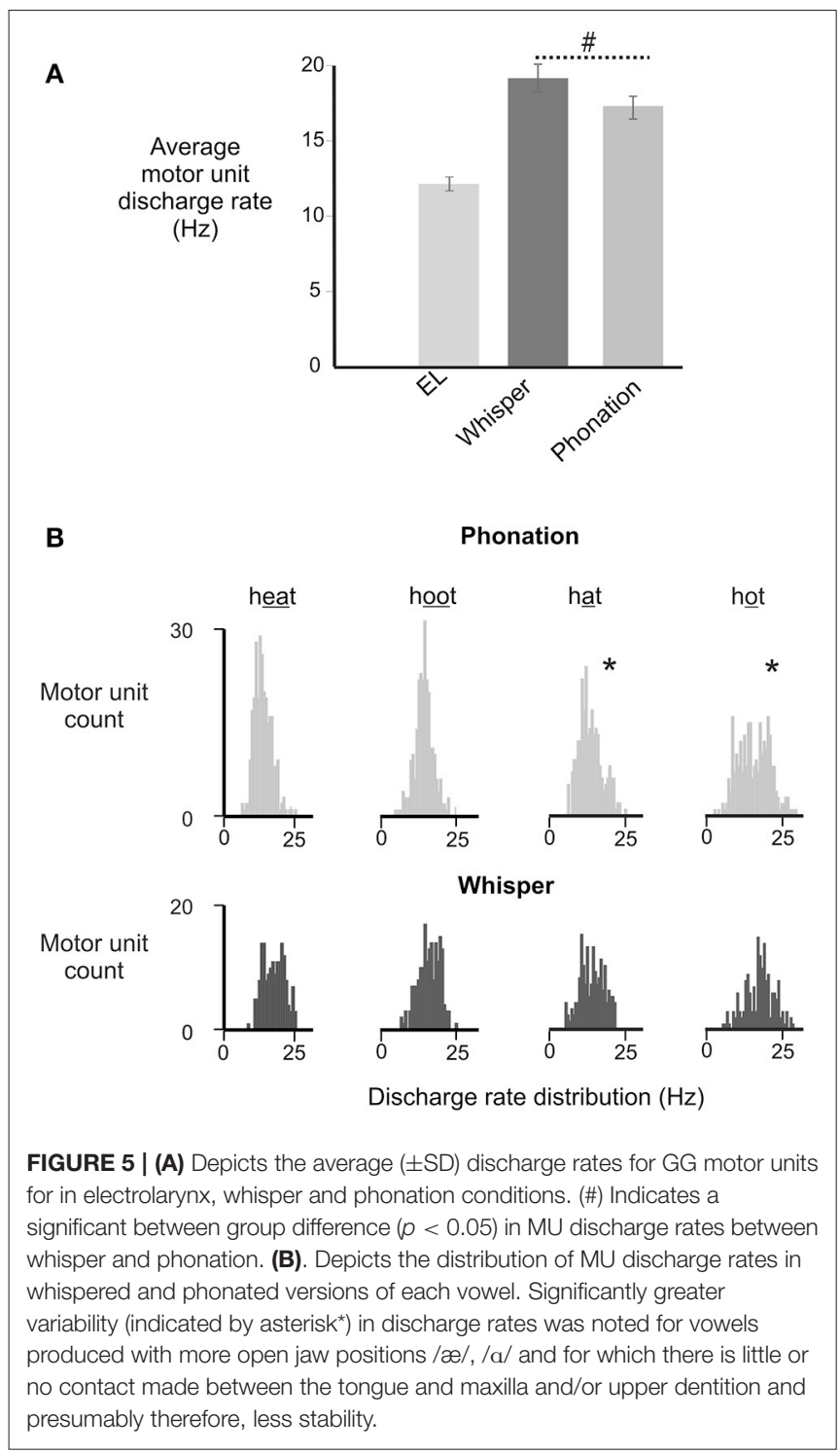

and phonated vowels were characterized by distinct patterns of activation, motor unit activity in the EL production of the same vowel shows no firing rate modulation.

There was a significant main effect of vocal condition $\left[F_{(1,168)}=4.52, p=0.035\right]$ with average motor unit discharge rates in whisper exceeding those in phonation. Interestingly, an interaction between sex and vocal condition approached significance $\left[F_{(1,168)}=3.59, p=0.06\right]$. That is, whisper was associated with higher average discharge rates in men than in women. However, in the absence of a significant effect the data were collapsed across male and female participants. For the group as a whole, motor unit discharge rates ranged from a minimum of $10.3 \mathrm{~Hz}$ to a maximum of $25.8 \mathrm{~Hz}$. Motor unit firing rates in phonation were below $20 \mathrm{~Hz}$ for the majority (26/31) of subjects whereas firing rates in whisper exceeded $20 \mathrm{~Hz}$ in $17 / 31$ subjects. In contrast, the lowest average firing rates were recorded in the electro-larynx condition with firing rates ranging between 12 and $14 \mathrm{~Hz}$.
The distribution of motor unit firing rates for all subjects in whisper and phonation are presented in Figure 5B. Although average motor unit discharge rate for $/ \mathrm{i} /, / \Re /, / \mathrm{u} /$, and $/ \mathrm{a} /$ were not different $\left[F_{(3,95)}=0.82, p=0.448\right]$, the discharge rate variances were different $\left[F_{(3,79)}=2.364, p<0.03\right]$. Specifically, the average firing rate was more variable for vowels produced with a more open mouth and lower tongue position ([æ] and [a]) (SD: 3.97 $\mathrm{Hz}$ ) relative to than vowels produced with a more closed mouth and correspondingly higher tongue position [(i) and (u) (SD: 2.79 $\mathrm{Hz})\left[F_{(1,50)}=7.257, p<0.01\right]$.

\section{DISCUSSION}

This study has three main findings. First, we provide evidence that the posterior region of the GG may contribute to changes in airway shape and stiffness in speech tasks that are performed exclusively on expiration. Second, we show that motor unit activity patterns in the EL condition are distinct from those in whisper and phonation. Thus, whereas motor unit activity persists throughout EL productions of each vowel, average motor unit firing rates decline and flow dependent modulation is eliminated. Last, in the switch from a low airflow condition (phonation) to a higher airflow condition (whisper), GG motor unit firing rates increase consistent with an increase in muscle activation. Given the GG's origin on the mandible, such activation likely results in forward motion of the tongue base and to expiration-related airway dilation.

\section{Experimental Method}

Although GG activity on expiration has been characterized previously (Sauerland and Mitchell, 1970; Sauerland and Harper, 1976; Saboisky et al., 2006, 2007) this aspect of the muscle's function has garnered much less attention. Recently, we recorded GG motor unit activity during moderate and heavy bicycling and noted that GG activation persisted throughout both the inspiratory and expiratory phases of each breath cycle (Walls et al., 2013). In light of the aforementioned, we sought alternative behavioral contexts in which to assess expiration-related GG activation. Speech, and sound production more broadly, is ideal in this regard because it entails volitional control of respiration (Loucks et al., 2007) and requires sustained expirations and because laryngeal and pharyngeal airway muscles participate in the regulation of the expiratory airstream. Using this approach, we were able to effect changes in laryngeal airway aperture simply by asking subjects to produce whispered or phonated versions of four vowels. We incorporated a third condition, the electrolarynx condition, to uncouple pharyngeal, and laryngeal airways while still operating within the framework of sound production. These manipulations provided a means of gauging how expirationrelated GG activity correlates with change/s imposed at the larynx.

\section{Regulation of Expiratory Airflow}

Previously published airflow rates for whisper encompass the range 0.2-0.9 L/s (Monoson and Zemlin, 1984; Stathopoulos et al., 1991; Sundberg et al., 2010) and for phonation, 0.08-0.17 L/s (Terasawa et al., 1987; Bailey and Hoit, 2002). Interestingly, 
these values are higher (whisper) and lower (phonation) than air flow rates reported during moderate-heavy exercise $0.25-$ $0.43 \mathrm{~L} / \mathrm{s}$ (Walls et al., 2013). However, a key distinction between speech/sound production and exercise lies in the lung volume excursions for the two tasks. In speech or sound production, lung volumes excursions typically encompass $\sim 400-500 \mathrm{ml}$ (Bailey and Hoit, 2002) as compared to $\sim 850 \mathrm{ml}$ in moderate and heavy exercise (Walls et al., 2013). This distinction is an important consideration because GG EMG is modulated by feedback from pulmonary stretch receptors (PSRs) (Brouillette and Thach, 1980; van Lunteren et al., 1984). PSRs are stimulated by lung inflation but in adults, the volume threshold for their activation is $\sim 1.5-$ 2.0 times the individual's resting tidal volume (Lind and Hesser, 1984). Whereas, tidal volume excursions of this magnitude are more common in heavy exercise and likely contribute to PSRrelated inhibition of GG EMG, conversational speech operates within the mid-range of lung volumes and thus, it is unlikely that PSR feedback is triggered in this context.

\section{Motor Unit Discharge}

Although there were no differences in motor unit firing rates between vowels, discharge rates varied more in the production of low and back vowels than in high and front vowels (Figure 5B). Differences in discharge rate variability previously have been attributed to differences in nervous system control-specifically the number of inputs that converge onto the motoneuron however, for the tongue this variability more likely is a function of the position of the jaw upon which the tongue rests (Shiller et al., 2002; Iskarous et al., 2011). As noted, GG motor unit discharge variability was lowest in production of the vowels /i/, and /u/ (i.e., heat and hoot) that are produced with a relatively closed mouth and with contact made between the tongue and the palate and/or teeth. Conversely, discharge variability was greatest for the vowels /æ/, /a/ (i.e., hat and hot) that are produced with a more open mouth and with no contact made between the tongue and palate and/or teeth. Thus, in the absence of a bony skeleton, the tongue's contact with an external bony target may confer much-needed stability (Gick et al., 2013).

Although we observed a trend toward somewhat higher average motor unit discharge rates in men than women, there

\section{REFERENCES}

Agostoni, E., Citterio, G., and D’Angelo, E. (1979). Decay rate of inspiratory muscle pressure during expiration in man. Respir. Physiol. 36, 269-285. doi: 10.1016/0034-5687(79)90041-0

Bailey, E. F., and Hoit, J. D. (2002). Speaking and breathing in high respiratory drive. J. Speech Lang. Hear. Res. 45, 89-99. doi: 10.1044/1092-4388(2002/007)

Bailey, E. F., Rice, A. D., and Fuglevand, A. J. (2007). Firing patterns of human genioglossus motor units during voluntary tongue movement. J. Neurophysiol. 97, 933-936. doi: 10.1152/jn.00737.2006

Bilodeau, M., Erb, M. D., Nichols, J. M., Joiner, K. L., and Weeks, J. B. (2001). Fatigue of elbow flexor muscles in younger and older adults. Muscle Nerve 24, 98-106. doi: 10.1002/1097-4598(200101)24:1<98::AID-MUS11>3.0.CO;2-D

Brouillette, R. T., and Thach, B. T. (1980). Control of genioglossus muscle inspiratory activity. J. Appl. Physiol. 49, 801-808.

Buchaillard, S., Perrier, P., and Payan, Y. (2009). A biomechanical model of cardinal vowel production: muscle activations and the impact of gravity on tongue positioning. J. Acoust. Soc. Am. 126, 2033-2051. doi: 10.1121/1.3204306 is very little data that supports the notion of sex-related differences in motor unit activation patterns. Whereas, several previous studies point to sex based differences in fatigability (Semmler et al., 1999; Bilodeau et al., 2001; Hunter et al., 2006), evidence of sex-based differences in motor unit firing rates are harder to find. To our knowledge, only one previous study (Christie and Kamen, 2010) noted differences in maximal motor unit discharges rates that were $\sim 9.0 \%$ higher in young men than in young women $(32.7 \pm 6.8 \mathrm{~Hz}$ vs. $29.3 \pm 7.0$ $\mathrm{Hz}, p=0.05)$. Nevertheless, in view of the unequal number of male $(N=10)$ and female $(N=21)$ participants in the current study, the possibility remains that the difference reported here is a function of non-physiological factors (Hunter, 2014).

\section{Summary}

We show increases in GG activation that occur against a background of changing airflow through the glottis. These findings are consistent with the notion of complimentary regulation of the laryngeal and pharyngeal airways (McClean and Tasko, 2002) and of the system wide regulation of airflow (Warren, 1986) but differ from previously published findings that show a diminution in expirationrelated GG activities during moderately heavy exercise (Walls et al., 2013).

\section{AUTHOR CONTRIBUTIONS}

AL: acquisition of EMG data, analysis and interpretation of data and approval of the submitted version. PW: conception and design, interpretation of data, writing of the manuscript and approval of the submitted version to be published. EB: conception and design, interpretation of data, reporting of the results, writing of the manuscript and final approval of the version to be published.

\section{ACKNOWLEDGMENTS}

This work was supported by a grant from the National Institute of Health (NIDCD 009587). 
Finnegan, E. M., Luschei, E. S., and Hoffman, H. T. (2000). Modulations in respiratory and laryngeal activity associated with changes in vocal intensity during speech. J. Speech Lang. Hear. Res. 43, 934-950. doi: 10.1044/jslhr.4304.934

Fogel, R. B., Malhotra, A., Pillar, G., Edwards, J. K., Beauregard, J., Shea, S. A., et al. (2001). Genioglossal activation in patients with obstructive sleep apnea versus control subjects. Mechanisms of muscle control. Am. J. Respir. Crit. Care Med. 164, 2025-2030. doi: 10.1164/ajrccm.164.11.2102048

Gick, B., Stavness, I., and Wilson, I. (2013). Speaking tongues are always braced. Jo. Acoust. Soc. Am. 134, 4204. doi: 10.1121/1.4831431

Giering, R. W., and Daubenspeck, J. A. (1990). Time course of laryngeal aperture response to expiratory resistance loading in humans. Respir. Physiol. 81, 371-379. doi: 10.1016/0034-5687(90)90117-H

Gilbert, R. J., Wedeen, V. J., Magnusson, L. H., Benner, T., Wang, R., Dai, G., et al. (2006). Three-dimensional myoarchitecture of the bovine tongue demonstrated by diffusion spectrum magnetic resonance imaging with tractography. Anat. Rec. A Discov. Mol. Cell. Evol. Biol. 288, 1173-1182. doi: 10.1002/ar.a.20387

Gillespie, A. I., Slivka, W., Atwood, C. W. Jr., and Verdolini Abbott, K. (2015). The effects of hyper- and hypocapnia on phonatory laryngeal airway resistance in Women. J. Speech Lang. Hear. Res. 58, 638-652. doi: 10.1044/2015_JSLHR-S-13-0270

Grimby, G., Bunn, J., and Mead, J. (1968). Relative contribution of rib cage and abdomen to ventilation during exercise. J. Appl. Physiol. 24, 159-166.

Hillenbrand, J., Getty, L. A., Clark, M. J., and Wheeler, K. (1995). Acoustic characteristics of American English vowels. J. Acoust. Soc. Am. 97, 3099-3111. doi: $10.1121 / 1.411872$

Hunter, S. K. (2014). Sex differences in human fatigability: mechanisms and insight to physiological responses. Acta Physiol. (Oxf). 210, 768-789. doi: 10.1111/apha.12234

Hunter, S. K., Butler, J. E., Todd, G., Gandevia, S. C., and Taylor, J. L. (2006). Supraspinal fatigue does not explain the sex difference in muscle fatigue of maximal contractions. J. Appl. Physiol. (1985) 101, 1036-1044. doi: 10.1152/japplphysiol.00103.2006

Iskarous, K., Shadle, C. H., and Proctor, M. I. (2011). Articulatory-acoustic kinematics: the production of American English /s. J. Acoust. Soc. Am. 129, 944-954. doi: 10.1121/1.3514537

Lind, F., and Hesser, C. M. (1984). Breathing pattern and occlusion pressure during moderate and heavy exercise. Acta Physiol. Scand. 122, 61-69. doi: 10.1111/j.1748-1716.1984.tb07482.x

Loucks, T. M., Poletto, C. J., Simonyan, K., Reynolds, C. L., and Ludlow, C. L. (2007). Human brain activation during phonation and exhalation: common volitional control for two upper airway functions. Neuroimage 36, 131-143. doi: 10.1016/j.neuroimage.2007.01.049

Matsuda, M., and Kasuya, H. (1999). Acoustic nature of the whisper. Eurospeech 99, 133-136.

McClean, M. D., and Tasko, S. M. (2002). Association of orofacial with laryngeal and respiratory motor output during speech. Exp. Brain Res. 146, 481-489. doi: 10.1007/s00221-002-1187-5

Mezzanotte, W. S., Tangel, D. J., and White, D. P. (1992). Waking genioglossal electromyogram in sleep apnea patients versus normal controls (a neuromuscular compensatory mechanism). J. Clin. Invest. 89, 1571-1579. doi: 10.1172/JCI115751

Mezzanotte, W. S., Tangel, D. J., and White, D. P. (1996). Influence of sleep onset on upper-airway muscle activity in apnea patients versus normal controls. Am. J. Respir. Crit. Care Med. 153, 1880-1887. doi: 10.1164/ajrccm.153.6.8665050

Miyawaki, K., Hirose, J., Ushijima, T., and Sawashima, M. (1975). A preliminary report on the electromyographic study of the activity of lingual muscles. RILP 9, 91-106.

Monoson, P., and Zemlin, W. R. (1984). Quantitative study of whisper. Folia Phoniatr. (Basel). 36, 53-65. doi: 10.1159/000265721

Peters, H. F., and Boves, L. (1988). Coordination of aerodynamic and phonatory processes in fluent speech utterances of stutterers. J. Speech Hear. Res. 31, 352-361. doi: 10.1044/jshr.3103.352

Peterson, G., and Barney, H. (1952). Control methods used in a study of the vowels. J. Acoust. Soc. Am. 24, 175-184. doi: 10.1121/1.1906875

Remmers, J. E. (2001). Wagging the tongue and guarding the airway. Reflex control of the genioglossus. Am. J. Respir. Crit. Care Med. 164, 2013-2014. doi: 10.1164/ajrccm.164.11.2110043a

Remmers, J. E., and Bartlett, D. Jr. (1977). Reflex control of expiratory airflow and duration. J. Appl. Physiol. 42, 80-87.
Remmers, J. E., deGroot, W. J., Sauerland, E. K., and Anch, A. M. (1978). Pathogenesis of upper airway occlusion during sleep. J. Appl. Physiol. 44, 931-938.

Saboisky, J. P., Butler, J. E., Fogel, R. B., Taylor, J. L., Trinder, J. A., White, D. P., et al. (2006). Tonic and phasic respiratory drives to human genioglossus motoneurons during breathing. J. Neurophysiol. 95, 2213-2221. doi: 10.1152/jn.00940.2005

Saboisky, J. P., Gorman, R. B., De Troyer, A., Gandevia, S. C., and Butler, J. E. (2007). Differential activation among five human inspiratory motoneuron pools during tidal breathing. J. Appl. Physiol. 102, 772-780. doi: 10.1152/japplphysiol.00683.2006

Sapienza, C. M., Stathopoulos, E. T., and Brown, W. S. Jr. (1997). Speech breathing during reading in women with vocal nodules. J. Voice 11, 195-201. doi: 10.1016/S0892-1997(97)80078-1

Sauerland, E. K., and Harper, R. M. (1976). The human tongue during sleep: electromyographic activity of the genioglossus muscle. Exp. Neurol. 51, 160-170. doi: 10.1016/0014-4886(76)90061-3

Sauerland, E. K., and Mitchell, S. P. (1970). Electromyographic activity of the human Genioglossus muscle in response to respiration and to positional changes of the head. Bull. Los Angeles Neurol. Soc. 35, 69-73.

Schwartz, M. F. (1968). Air consumption, per syllable, in oral and whispered speech. J. Acoust. Soc. Am. 43, 1448-1449. doi: 10.1121/1.1911007

Seashore, C. E. (1938). Psychology of Music. North Chelmsford, MA: Courier Corporation.

Semmler, J. G., Kutzscher, D. V., and Enoka, R. M. (1999). Gender differences in the fatigability of human skeletal muscle. J. Neurophysiol. 82, 3590-3593.

Shiller, D. M., Laboissiere, R., and Ostry, D. J. (2002). Relationship between jaw stiffness and kinematic variability in speech. J. Neurophysiol. 88, 2329-2340. doi: $10.1152 /$ jn. 00286.2002

Simonyan, K., and Horwitz, B. (2011). Laryngeal motor cortex and control of speech in humans. Neuroscientist 17, 197-208. doi: 10.1177/1073858410386727

Solomon, N. P., McCall, G. N., Trosset, M. W., and Gray, W. C. (1989). Laryngeal configuration and constriction during two types of whispering. J. Speech Hear. Res. 32, 161-174. doi: 10.1044/jshr.3201.161

Stathopoulos, E. T., Hoit, J. D., Hixon, T. J., Watson, P. J., and Solomon, N. P. (1991). Respiratory and laryngeal function during whispering. J. Speech Hear. Res. 34, 761-767. doi: 10.1044/jshr.3404.761

Sundberg, J., Scherer, R., Hess, M., and Muller, F. (2010). Whispering-a singlesubject study of glottal configuration and aerodynamics. J. Voice 24, 574-584. doi: 10.1016/j.jvoice.2009.01.001

Terasawa, R., Hibi, S. R., and Hirano, M. (1987). Mean airflow rates during phonation over a comfortable duration and maximum sustained phonation. Results from 60 normal adult subjects. Folia Phoniatr. 39, 87-89. doi: $10.1159 / 000265843$

van Lunteren, E., Strohl, K. P., Parker, D. M., Bruce, E. N., Van de Graaff, W. B., and Cherniack, N. S. (1984). Phasic volume-related feedback on upper airway muscle activity. J. Appl. Physiol. 56, 730-736.

Walls, C. E., Laine, C. M., Kidder, I. J., and Bailey, E. F. (2013). Human hypoglossal motor unit activities in exercise. J. Physiol. (Lond). 591, 3579-3590. doi: 10.1113/jphysiol.2013.252452

Warren, D. W. (1986). Compensatory speech behaviors in individuals with cleft palate: a regulation/control phenomenon? Cleft Palate J. 23, 251-260.

Warren, D. W., Morr, K. E., Rochet, A. P., and Dalston, R. M. (1989). Respiratory response to a decrease in velopharyngeal resistance. J. Acoust. Soc. Am. 86, 917-924. doi: 10.1121/1.398726

Weismer, G., and Longstreth, D. (1980). Segmental gestures at the laryngeal level in whispered speech: evidence from an aerodynamic study. J. Speech Hear. Res. 23, 383-392. doi: 10.1044/jshr.2302.383

Conflict of Interest Statement: The authors declare that the research was conducted in the absence of any commercial or financial relationships that could be construed as a potential conflict of interest.

Copyright (c) 2017 LaCross, Watson and Bailey. This is an open-access article distributed under the terms of the Creative Commons Attribution License (CC BY). The use, distribution or reproduction in other forums is permitted, provided the original author(s) or licensor are credited and that the original publication in this journal is cited, in accordance with accepted academic practice. No use, distribution or reproduction is permitted which does not comply with these terms. 